\title{
Characteristics Analysis for Volatile Organic Compounds Emission of Wood Furniture
}

\author{
Miao Yanfeng, Guan Huiyuan*, Chen Yulong \\ College of Furniture and Industrial Design, Nanjing Forestry University, Nanjing, China
}

\begin{abstract}
Man-made interior decoration board is a kind of common porous material, which is made of solid skeleton with fluid inside the complex mixture. The establishment of the interior decoration multiphase multidimensional model release by artificial board, is based on the geometrical characteristic of wood panel thickness. The artificial board TVOC release by multiphase multidimensional model of mass transfer is simplified into a one-dimensional heterogeneous mass transfer model, and the solution and numerical simulation was also a one-dimensional model. This paper investigates a man-made board to release characteristics of volatile organic pollutants from decoration. By theoretical analysis and experimental methods, artificial board TVOC release was discussed. The finite element method is introduced into the furniture structural mechanics research, and the study of the computer finite element simulation analysis was applied for the determination of furniture structure, which designed mechanical properties of the theoretical foundation, system construction and concrete methods.
\end{abstract}

Keywords: Multiphase mass transfer model, release characteristics, volatile organic compounds, wood-based panel.

\section{INTRODUCTION}

People pay more attention to the social awareness of environmental protection and volatile organic compounds used for maintaining indoor air quality [1-3]. The use of a large number of man-made board room decoration was the main source of TVOC. In order to improve indoor air quality, management of indoor TVOCs, the release mechanism and the influence factors of wood-based panels that are fully integrated to the TVOC are discussed. This paper studies the interior decoration of the common market for artificial board TVOC release mechanism and influence factors [4]. The establishment of artificial board TVOC release multiphase mass transfer model; analysis of environmental factors such as temperature, relative humidity, effect the board TVOC release and characteristics; finally, the TVOC prediction model and method is discussed [5].

Indoor air pollution has become a serious problem and it promotes the development of VOC control measures, which are three main ways: source control, ventilation and air purification [6,7]. The source control of the most potential governance is the most effective, which can not only avoid mechanical ventilation energy consumption, but also can avoid the problem of air purification by-products. A fundamental method put things right once and for all. The governments and trade associations have been established which fit the situation of our country health standards, which support the provisions of the main VOC indoor environment and building materials in the limit of concentration of VOC emission limit [8-12].

*Address correspondence to this author at the College of Furniture and Industrial Design, Nanjing Forestry University, Nanjing, China; E-mail:myf1203@163.com
Understanding the release mechanism of interior decoration materials has important significance for the study of indoor air quality and pollution problems. Diffusion and transformation of pollutants in the environment is an important subject in scientific research of environmental science and engineering. This paper intends to analyze the mechanism of diffusion of volatile organic pollutants in porous building materials and indoor air from the point of view of porous media. The porous materials was the research object to explore the particleboard; therefore, influence of environmental conditions on the release of volatile organic pollutants in particleboard, its theoretical basis and scientific method for the effective control of man-made board caused by indoor VOC pollution were discussed [13-16].

Finite element method solves the structure stress analysis problems applicably, which is widely used in many complex structure based on strength, stiffness, stability analyses and calculations. It has thus become the most effective method in structure analysis field. With the development of computer technology, finite element method for computer simulation analysis becomes the product design performance analysis and simulation which is a very effective method. This study is based on the in-depth systematic study of the finite element method, computer simulation technology research, and solid wood frame type furniture structural mechanics.

\section{RELATED METHOD AND THEORY}

\section{Research on Dynamic TVOC Release}

Theoretical study is established based on mass transfer theory, which is widely known as the mechanism model and physical model. Each parameter has its fixed physical meaning. The establishment of mechanism model has very important significance for the research of release mechanism of TVOC during building materials $[17,18]$. 
The model established based on VBX model, TVOC model: describes the diffusion of classic; the model ignores the internal diffusion resistance; and the concentration of TVOC assumed material film is uniform [19].

$R=h_{m}\left(\frac{p_{\gamma} M}{M_{0}}-C_{\infty}\right)$

$\mathrm{R}$ : release rate;

$\mathrm{h}_{\mathrm{m}}$ : convection mass transfer coefficient;

M: TVOC the amount of material remaining

$\mathrm{P}_{\mathrm{T}}$ : The initial steam pressure.

So the model can be used only with the control action on evaporation process [20].

Researcher established analytical model of TVOC with dry material distribution. Xu and Zhang improved the model, such that the control equation of material, material air interface and the air side in the diffusion model, are as follows:

$\frac{\partial C_{m}}{\partial t}=D \frac{\partial^{2} C_{m}}{\partial x^{2}}$

$-D_{x} \frac{\partial C_{m}}{\partial x}=h_{m}\left(C_{a N}-C\right), \quad x=l, 0 \leq t \leq T$

$V_{c} \frac{d C(t)}{d t}=A_{m} h_{m}\left(C_{a N}-C\right)$

This material is the diffusion coefficient D, CM shows the material of internal TVOC concentration. They carried out numerical simulation of dry material to study the impact of initial concentration, the internal diffusion coefficient, separation factor and material life that effect release of material TVOC [21].

\section{Commonly Used Physical Quantities in Porous Media}

The porous medium assumed a connected pipe network; in this model, each tube is fixed space flow tube with the same geometry. The concept of curvature is the piezometric head by Carman at an average rate bent in a capillary tube. Suppose, the length of the pipe is connected at both ends with the line length, the direction of the line was called, it defined the curvature in the direction of $\mathrm{L} / \mathrm{La}$. The porous media is bending at a rate of rank two tensor, denoted as follows [22]:

$T=\left(\begin{array}{lll}T_{11} & T_{12} & T_{13} \\ T_{21} & T_{22} & T_{23} \\ T_{31} & T_{32} & T_{33}\end{array}\right)$

For isotropic medium, the curvature tensor can be simplified to a simple scalar, and hence the Baer in the dynamics of fluids in porous media has been proved. Experience numerical curve rate was 0.71 , i.e. the average curvature values given in the literature changed in the range of 0.56 to 0.8 [23].

Because the porous material is a multiphase mixture, its density is composed of solid skeleton and multicomponent gas decision. That is if the porosity is $\varepsilon$, the solid skeleton and the density of the gas is respectively $\rho d$ and $\rho$, and the average density of $\rho$ is:

$$
\bar{\rho}=\rho_{d}(1-\varepsilon)+\rho_{a} \varepsilon
$$

The Establishment of Multiphase Mass Transfer Model

TVOC release from artificial board can be divided into three processes: namely the internal sheet, plate interface and release sheet [24], which was shown in Fig. (1):

The area of four sides of artificial plate relative to the plane area, is very small. In addition, the experimental study often leads to edge treatment. Four parts of hypothesis cover non-volatile organic pollutants, man-made board release and a double-sided board release.

The building is placed between two identical modules, which is shown in Fig. (2); a glass chamber with a constant concentration of VOC, while the other glass chamber with clean air; the concentration at the outlet of two cabins is the hourly record. When the mass transfer process reaches a steady state, the diffusion law and diffusion coefficient of Dm can be calculated by the following formula:

$D=-\frac{m}{A} \frac{\Delta x}{\Delta c}=\frac{Q L}{A} \frac{C_{2}}{C_{1}-C_{2}}$

The traditional method of furniture design does not provide a true analysis of furniture. İt can only be used as a simplified model for analysis. Because it is difficult to know the full potential of a real furniture in the process of various components design, they are usually simplified into an imaginary furniture model to analyze. Based on the assumption of a simplified model of the furniture parts, a node is completely free to rotate in the complex situation, all components and nodes work as a whole in case the deformation of its structure is considered, as long as the ideal structural properties and real structure are similar. Except for the special precise design, this analysis may be applied for designing practical furniture.

In the furniture structure aided optimization design, furniture structure model system integrating software is used for analysing the material's properties. Finite element software analysis module is composed of a furniture structure and mechanical strength of aided design and determination of evaluation of the collected system. Software system needs to be built through the material testing and solid modeling, to establish material parameter database and furniture entity models library. The property parameter of material and furniture solid model correspond to ANSYS finite element analysis software simulation; calculations and the results were analyzed according to the optimized design.

\section{EXPERIMENTAL RESULTS}

\section{Test Apparatus and İnstruments}

The sealed environmental chamber material is shown in Fig. (3). In order to reduce the adsorption/desorption effect of VOC gas, the entire cabin was made with stainless steel, and the environmental chamber wall received polished treatment, in compliance with the ASTM standard. The 


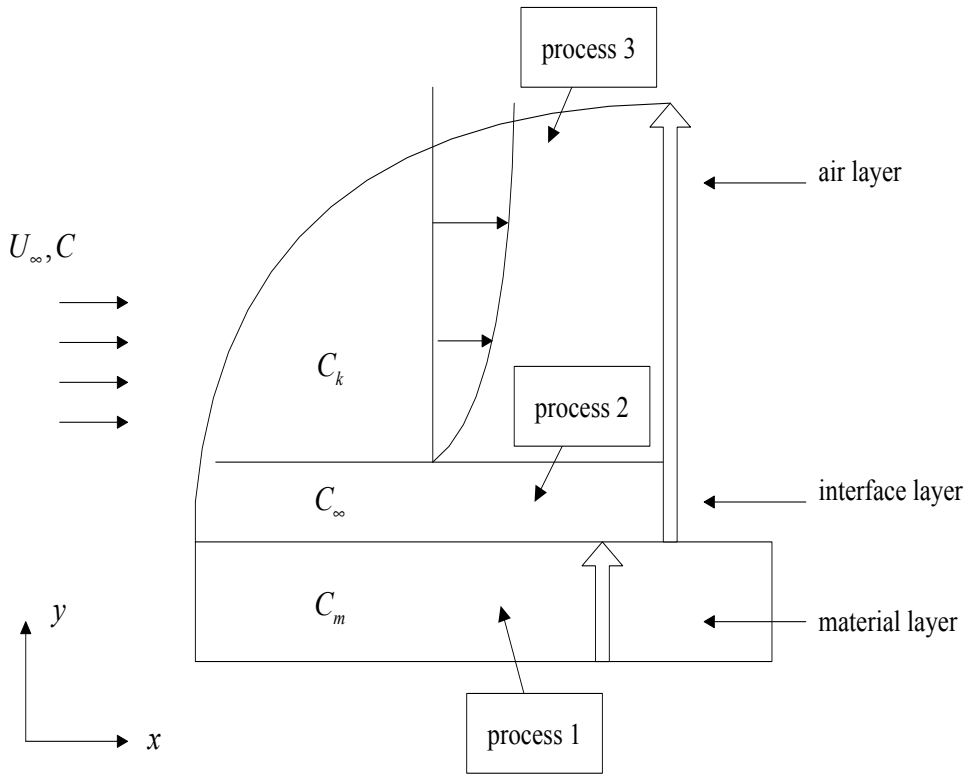

Fig. (1). TVOC release process in artificial board.

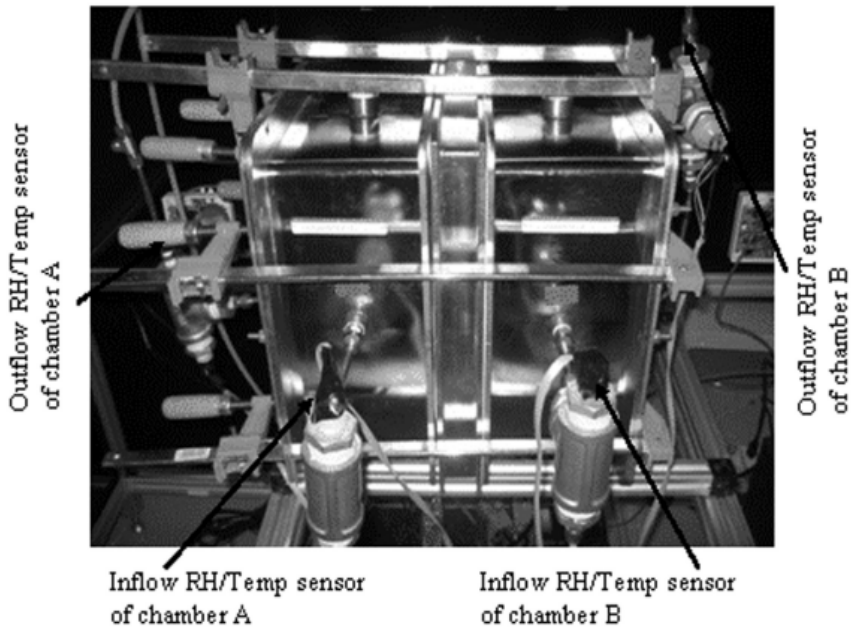

Fig. (2). The building placed between two identical modules.

diameter of sealed environmental chamber was $300 \mathrm{~mm}$, was $430 \mathrm{~mm}$ high, the cavity volume $\mathrm{w}$ was $30 \mathrm{~L}$; environmental chamber was at the top of a DC fan, to produce a similar indoor mobile environment, and make the cabin VOC mixing.

From analysis of the above shelf of deformation and stress, it is found that the deformation is smaller than the required amount of deformation with provisions standard, each component stress is within the stress limits, which can satisfy the design usage requirement reasonably, but it can support the bookcase pieces of shelf in joint stress concentration more seriously through the support piece connection position optimization. This design can improve the stress state, making the design more reasonable and prolong service life.

Efficient use of wood resources plays an important role in alleviating the contradiction between timber supply and demand. Thus, promoting the rise and development of furniture, helps mainstream products on the market. With the development of society and progress of science and technology, people's demand of high quality furniture products is strongly increased. Although structural strength of furniture products design is increasing furniture production and research departments, the structural strength of furniture design also requires the continuous development and improvement. This paper used the finite element theory, ANSYS analysis software and optimization design technology. Through the theoretical analysis, computer simulation, and experimental research and optimal design; structure of plate furniture, connection mode and connection position was achieved.

\section{The Analysis of Results}

This paper mainly studied the formaldehyde density board as the target pollutant, as formaldehyde gas detection was close to the environmental compartments. 
Formaldehyde gas is injected into the empty cabin without building materials in the sealed chamber, which is recorded by formaldehyde concentration decay curve. If the cabin wall surface adsorption is not considered, the concentration of formaldehyde in the cabin environment can be described by the following equation:

$V \frac{d C_{a}(t)}{d t}=Q\left[C_{i n}-C_{a}(t)\right]$

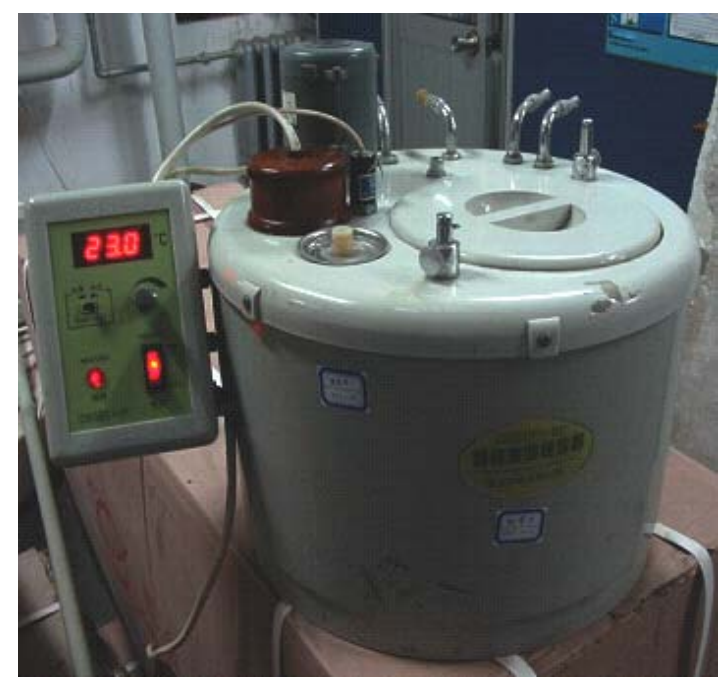

Fig. (3). The sealed environmental chamber material.

The environmental chamber is generally slow and uniform, and the impact of formaldehyde concentration on the outside leakage could not be traced; so it can be concluded that $\mathrm{Q}$ and $\mathrm{Cm}$ are constants, which can get the solution as per the following equation:

$\ln \left[C_{a}(t)-C_{i n}\right]=-\frac{Q}{V} \cdot t+\ln \left(C_{1}-C_{i n}\right)$

On the measured attenuation curve of formaldehyde in the cabin environment, the types of slope are in the form of linear fitting. Fig. (4) shows tightness detection results of the test at a temperature of $25{ }^{\circ} \mathrm{C}$; it can be seen that the hourly air leakage is only $0.35 \%$ of the environmental chamber volume; the air leakage is compared with the effect of building materials distribution process; the VOC concentration in the cabin environment can be ignored.

Fig. (5) shows the variation curve with the concentration of formaldehyde by monitoring the cabin environment.

The detailed structure of solid model establishment based on each piece of model, is the fundamental research objective and was based on the development of scale analysis. In the main frame structure, furniture is the main body of scale and proportion. The shapes and sizes of main components are maximally truly consistent. In addition, component bonding form can be measured for a specific connection form and scale in accordance with the actual measured value modeling, which cannot be measured according to the similar bonding and birch joint technical requirements modeling. In the structure of modern furniture, there is very little use of joint, according to the modern form of processing which is appropriately simplified.

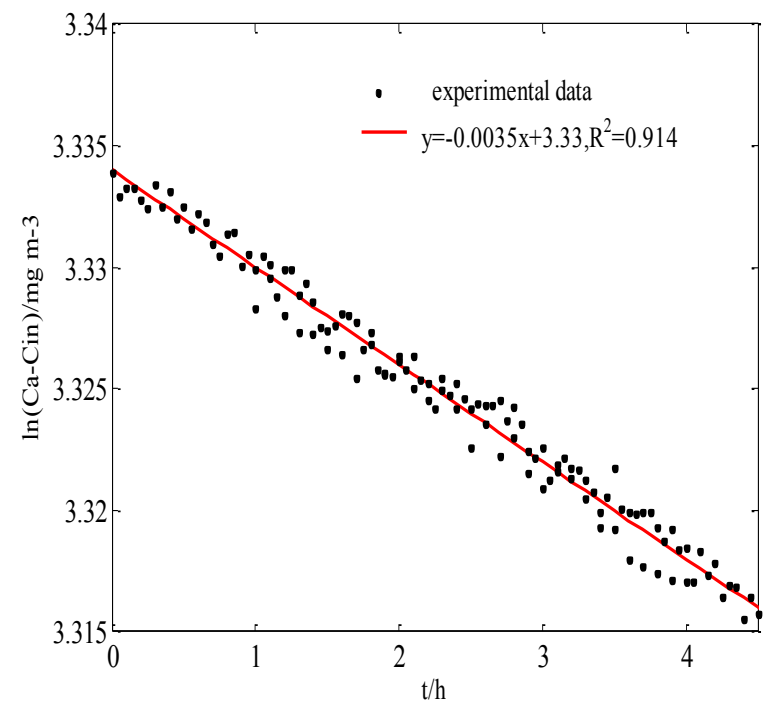

Fig. (4). Tightness detection results of the test at a temperature of $25{ }^{\circ} \mathrm{C}$.

Traditional furniture component form and joint changes have taken many molds, carvings and other decorative elements. Our model however, is in compliance with the real body needs due to spending a lot of modeling time. Too much camber line element during the ANSYS software processing and grid division will bring larger problems, which may also affect software operation speed and accuracy. If the furniture structural mechanics analysis would be typically structure and component based, contributing little to the structural strength and even unrelated elements can be simplified, it would not be possible to make the research more universal and representative.

\section{CONCLUSION}

This paper focuses on the use of wood-based panel decoration, and volatile organic matter release properties were studied. The theoretical analysis and experimental methods of man-made board TVOC release mechanism and release characteristics were discussed. According to the properties of porous artificial plate embedded with interior decoration, the interior is described as the solid skeleton with fluid inside the complex mixture, based on the continuum hypothesis. We established the interior decoration multiphase multidimensional model of mass transfer, i.e. volatile organic pollutants released by the board. As the artificial plate thickness is thin, the TVOC concentration gradient is parallel to the direction of thickness. In the direction of VOCs in artificial board release multiphase multidimensional model, mass transfer is simplified to a onedimensional heterogeneous mass transfer model, and the solution of the problme is one-dimensional model and numerical simulation. 

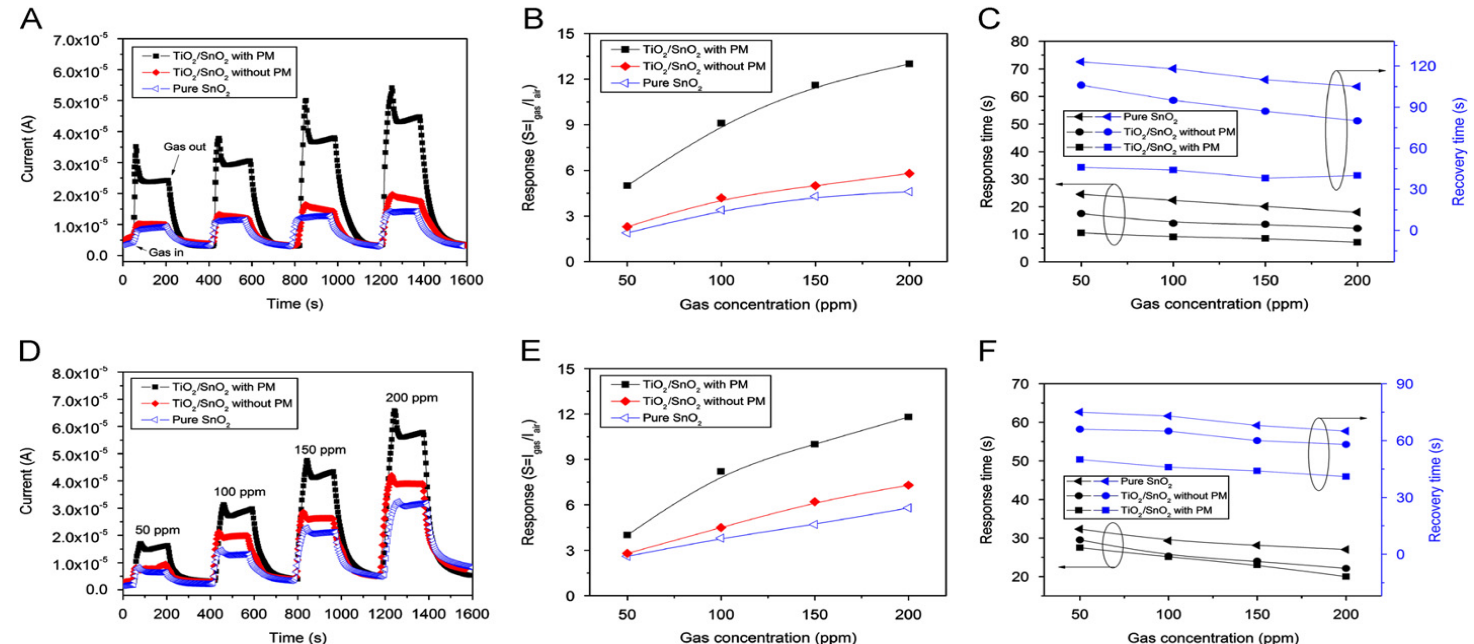

Fig. (5). The variation curve with different concentrations of formaldehyde.

\section{CONFLICT OF INTEREST}

The authors confirm that this article content has no conflict of interest.

\section{ACKNOWLEDGEMENTS}

The Priority Academic Program Development of Jiangsu Higher Education Institutions (PAPD) is highly acknowledged for support of this study.

\section{REFERENCES}

[1] J M Xin, SA. Linear prediction approach to direction estimation of cyclostationary signals in multipath environment. IEEE Transactions on Signal Processing 2001; 49(4): 710-20. [2] E Grosicki, K Abed-Meraim, K Y Hua. A weighted linear
prediction method for near-field source localization. IEEE Transactions on Signal Processing 2005; 53(10): 3651-60.

[3] T B Lavate, V K Kokate, A M Sapkal. Performance analysis of MUSIC and ESPRIT DOA estimation algorithms for adaptive array smart antenna in mobile communication. Inter J Comp Net 2010; 2(3): $152-8$

[4] A Hirata, T Morimoto, $\mathrm{Z}$ Kawasaki. DOA estimation of ultawideband EM waves with MUSIC and interferometry. IEEE Antennas and Wireless Propagation Letters 2003; 2(1): 190-3.

[5] F Taga. Smart Music algorithm for DOA estimation. Electronics Letters 1997; 33(3): 190-1.

[6] W Sun, J L Bai, K Wang. Novel method of ordinal bearing estimation for more sources based on oblique projector. Journal of Systems Engineering and Electronics 2009; 20(3): 445-9.

[7] Man Cheol Kim, Jinkyun Park, Wondea Jung, Hanjeom Kim, Yoon Joong Kim. Development of a standard communication protocol for an emergency situation management in nuclear power plants [J]. Annals of Nuclear Energy, 2010, 37(6): 888-893Qi S X, Ma J, Li H, Zhang S, et al. Infrared small target enhancement via phase spectrum of Quaternion Fourier Transform, Infrared Physics \& Technology. 62 (2014) 50-58.

[8] Barnett J. Statistical analysis of median subtraction filtering with application to point target detection in infrared backgrounds, Proceedings of the SPIE 1050 (1989) 10-15.

[9] Tom V T. Morphology-based algorithm for point target detection in infrared backgrounds, Proceedings of the SPIE 1954 (1993) 2-11.
[10] Deshpande S D. Max-mean and max-median filters for detection of small-targets, Proceedings of the SPIE 3809 (1999) 74-83.

[11] Kawi S, Tang YP, Hidajat K, Yu L E. Synthesis and characterization of nanoscale $\mathrm{CeO} 2$ catalyst for deNOx. J. Metastab Nanocryst Mater 2005; 23: 95-8.

[12] Llinas J. Hall, D.L. An Introduction to Multi-Sensor Data Fusion. In Proceedings of the 1998 IEEE International Symposium on Circuits and Systems (ISCAS 1998), Monterey, CA, USA, 31 May-3 June 1998; 537-40.

[13] Mao SY, Zhao W. Comments on multisensor image fusion techniques. J Beijing Univ Aeronaut Astronaut 2002; 28: 512-8.

[14] Di Gennaro, E Gallina, I Andreone, A Castaldi, G, Galdi V. Experimental evidence of cut-wire-induced enhanced transmission of transverse-electric fields through sub-wavelength slits in a thin metallic screen. Opt Express 2010; 18: 26769-74.

[15] Schurig D, Mock JJ, Justice BJ, Cummer SA, Pendry JB, Starr AF, Smith, DR. Metamaterial electromagnetic cloak at microwave frequencies. Science 2006; 314: 977-80.

[16] Christ A, Martin OJF, Ekinci Y, Gippius NA, Tikhodeev SG. Symmetry breaking in a plasmonic metamaterial at optical wavelength. Nano Lett 2008; 8: 2171-5.

[17] Papasimakis N, Fu YH, Fedotov FA, Prosvirnin SL, Tsai DP, Zheludev NI. Metamaterial with polarization and direction insensitive resonant transmission response mimicking electromagnetically induced transparency. Appl Phys Lett 2009; 94: 211902-4

[18] Singh R., Al-Naib IAI, Koch M, Zhang W. Sharp Fano resonances in THz metamaterials. Opt Express 2011; 19: 6314-9.

[19] Cao W, Singh R, Al-Naib I AI, He M, Taylor AJ, Zhang, W. Lowloss ultra-high-Q dark mode plasmonic Fano metamaterials. Opt Lett 2012; 37: 3366-8.

[20] Casasent D, Ye A. Detection filters and algorithm fusion for ATR, IEE. Transactions on Image Processing 1997; 6:114-25.

[21] Wang X, Liu L, Tang ZM. Infrared dim target detection based on fractal dimension and third-order characterization. Chinese Optics Letters 2009; 7:931-3.

[22] Sun S G. Target detection using local fuzzy thresholding and binary template matching in forward-looking infrared images. Optical Engineering 2007; 46: 036402.

[23] Itti L, Koch C, Niebur E. A model of saliency-based visual attention for rapid scene analysis, IEEE Trans. Pattern Anal Mach Intell 1998; 1254-1259.

[24] Ko B C, Nam J. Object-of-interest image segmentation based on human attention and semantic region clustering, J Opt Soc Am A $2006 ; 2462-70$. 